\title{
Differences between the motion of a helical vortex and the movement of fluid particles
} along its axis

Okulov, V.L.; Gesheva, E.S.; Kuibin, P.A.; Shtork, S.I.; Sørensen, J.; Wood, D.; Alekseenko, S.V.

Published in:

Thermophysics and Aeromechanics

Link to article, DOI:

$10.1134 /$ S0869864320040010

Publication date:

2020

Document Version

Peer reviewed version

Link back to DTU Orbit

Citation (APA):

Okulov, V. L., Gesheva, E. S., Kuibin, P. A., Shtork, S. I., Sørensen, J., Wood, D., \& Alekseenko, S. V. (2020).

Differences between the motion of a helical vortex and the movement of fluid particles along its axis.

Thermophysics and Aeromechanics, 27(4), 473-480. https://doi.org/10.1134/S0869864320040010

\section{General rights}

Copyright and moral rights for the publications made accessible in the public portal are retained by the authors and/or other copyright owners and it is a condition of accessing publications that users recognise and abide by the legal requirements associated with these rights.

- Users may download and print one copy of any publication from the public portal for the purpose of private study or research.

- You may not further distribute the material or use it for any profit-making activity or commercial gain

- You may freely distribute the URL identifying the publication in the public portal 

and the movement of fluid particles along its axis* D. Wood ${ }^{1,4}$, and S.V. Alekseenko ${ }^{2}$ ${ }^{1}$ Novosibirsk State University, Novosibirsk, Russia ${ }^{2}$ Kutateladze Institute of Thermophysics SB RAS, Novosibirsk, Russia ${ }^{3}$ Technical University of Denmark, Lyngby, Denmark

${ }^{4}$ Schulich school of Engineering, University of Calgary, Calgary, Canada

E-mail: vaok@dtu.dk; vokulov@mail.ru

(Received February 3, 2020; revised February 9, 2020; accepted for publication February 10, 2020)

The motion of a helical vortex and the movement of the fluid particles along its axis are analyzed. The same form of the helical axis of the vortex and the trajectory of the particles sometimes leads to a false assumption of equality of these two different motions. The correct identification of both motions, however, is essential when considering the helical vortices in the wakes of rotors, or the displacement of the core of helicoidal tornado, etc. Only the same helical shape is the vortex axis and the streamline of the fluid particles can mistakenly merge these two different motions as the identical travel. We describe an experiment that clearly separates these two motions for the case of a stationary helical vortex, along the axis of which the fluid particles intensively move. The result shows that the absolute velocity of fluid particles does not coincide with the motion of the helical vortex as a whole.

Keywords: vortex dynamics, helical vortex.

\section{Introduction}

The development of the theory of helical vortices has a long history, starting with the famous work of Lord Kelvin of 1880 on spiral perturbations of a column-shaped vortex, for the first time considering a large-pitch helical vortex [1]. Helical vortices are one of the main states of swirling flows, so they are of fundamental importance for fluid mechanics. Examples of vortex structures with a helical shape are well known in the wakes of rotors, concentrated vortices in vortex furnaces and burners, tornadoes, eddies in fluids, etc. It is necessary to correctly

\footnotetext{
* The work, including measurements of velocity fields and theoretical analysis, was carried out with the state support of scientific research, conducted under the guidance of leading scientists in Russian universities (agreement No. 07515-2019-1923). The visualization experiment was performed under the state contract with IT SB RAS (AAAA-A17117030910025-7).
}

(C) V.L. Okulov, E.S. Gesheva, P.A. Kuibin, S.I. Shtork ${ }^{1}$ J. Sørensen, D. Wood, and S.V. Alekseenko, 2020 
predict the dynamics of such structures, since vortex motion contributes to the intensification of heat and mass transfer and affects the performance of rotary machines. In turn, their stationary position provides stable operating modes in vortex devices. Currently, there is an increased interest in studying the interaction of helical vortices in the wakes of multiple turbines, in wind and hydro farms $[2,3]$. Determining the motion of helical vortices occupies an important place both in fluid mechanics and in aerodynamics and their applications, so many specialists in hydro-mechanics have been engaged in solving it (see, for example, works [4-8] and references to original research therein). In addition, the study of motion of helical vortices, being a special case of three-dimensional concentrated vortex filaments, is of interest from the point of view of determining the fundamental laws of their dynamics.

The derivation of the equation of motion of a three-dimensional vortex filament is associated with the work of Da Rios [9]. However, the authors of [10] show that the equations of motion in the special case of a helical vortex were also independently obtained by Joukowsky [4]. The result of both studies concludes that three-dimensional vortex filaments, including helical ones, move only in the binormal direction, while the tangential motion of particles along the vortex axis does not change the position in space of the vortex filaments. Subsequently, the derivation of the equations of dynamics of three-dimensional vortex filaments was repeated by many authors, and the proof of the binormal motion was presented in the book of Batchelor [11] (p.510). However, 50 years after the publication of this fundamental textbook, $[12,13]$ rejected the traditional description of the motion of helical vortices [4-11], but the classical description of [11] was explained again with different velocity polygons for the motions of a helical vortex and particles along its axis in a flat representation in Fig. 1 in [14].

Our view is that [12] states without justification that "The velocity of a helical vortex is represented as the sum of either the circumferential and axial components or the tangential and binormal components". Further, the equations of motion of the helical vortex (2.8) and (2.9) in [12], uses the absolute motion including the tangential motion that does not change the spatial position of the vortex, as indicated in [4]. The article [13], devoted to the motion of several helical vortices does not contain new ideas, since the well-known formula from [8] is used to estimate the contribution of additional vortices to the motion of the calculated vortex. Accordingly, [13] does not evoke new critical comments in addition to those mentioned above, except that it uses an erroneous formula from [12] associated with the speed of absolute movement of fluid particles, rather than with binormal motion of the helical vortex, to describe the vortex motion. In this paper, we describe an experiment to clearly demonstrate the difference between these two motions for the special case when the vortex is stationary, whereas the fluid particles along its axis have non-zero velocities.

\section{Experimental verification of the difference in the motion of vortex and particles}

The confusion in determining the velocity of a three-dimensional structurein $[12,13]$ is due to the fact that the absolute motion of particles along the axis and the binormal motion of the helix itself fix the same new position of the vortex axis in space (see Fig. 1 in [14]). The binormal displacement determines the shortest distance to the new axis position, and the absolute displacement defines the new position of the fluid particle on the displaced axis. Both motions coincide only for a plane vortex, when the vortex motion in the plane coincides with the movement of the fluid particles on the axis. However, in the three-dimensional case, fluid particles can move freely along the axis of the vortex without altering its position and shape. This internal motion of particles that does not change the shape of the structure must be excluded; otherwise an incorrect conclusion about the motion of the structure itself may be made. This can be clearly demonstrated in the extreme case when the three-dimensional vortex is stationary (Fig. 1) as visualized in a hydraulic model of a vortex combustion chamber (Fig. 1a). The details of the chamber can be found in $[15,16]$. A stationary helical vortex with significant motion of the fluid particles along its fixed axis is observed in the vortex chamber. In this case, 
the tangential motion is directed only along the axis and no axis shift in space occurs, as there is no velocity component in the binormal direction (Fig. $1 b$ ). Since any curved vortex has a self-induced speed in the binormal direction, the existence of stationary helical vortices is explained by the presence of an additional velocity that is induced by the wall of the vortex chamber and compensates for the self-induced motion (Fig. 1c). At the same time, consideration of the helical vortex in the $z, \theta$-plane demonstrates that the possible displacement of the axis of the helical vortex is uniquely determined by the binormal motion.

Next, we demonstrate that a stationary helical vortex (Fig. 1a) can exist with the significant motion of vortex particles along its axis, i.e. their absolute velocity cannot be equated to that of the structure itself. For this purpose, in addition to visual observations [15, 16], the authors measured the velocity fields using standard particle image velocimetry (PIV) techniques with the parameters given in [17]. Velocity fields were measured in several vertical planes that intersect the vortex axis. It is determined that the velocity of fluid particles at these points (Fig. 2b) coincides with the tangential direction along the axis of the vortex (Fig. 2a), whose angle with the horizontal plane corresponds to the helical pitch, determined from visual observations. Additional measurements were conducted in several horizontal sections that intersect the axis at points A and B, as shown in Fig. $2 a$, to confirm the stationarity of the helical vortex. The position of the vortex axis-is unchanged with time, including at the tested points A and B. This also follows from Fig. 3, showing the intersection points of the vortex axis with the horizontal plane at the test points A and B (Fig. 2a) at different arbitrary moments of time.

Analyzing data of Figs. 2 and 3, it can be concluded that the fluid particles move along the vortex axis with tangential, but no binormal velocity. Thus, the stationarity of the helical vortex (Fig. 1a) is explained by the absence of a binormal velocity in full accordance with the dynamics of three-dimensional vortex filament [11]. This means that the absolute motion of the vortex particles cannot indicate the motion of the helix. Only the velocity along the binormal direction, rather than the full absolute velocity, is responsible for this motion, and the tangential component for this description must be removed, as indicated by the authors of $[4-11,14]$.

106
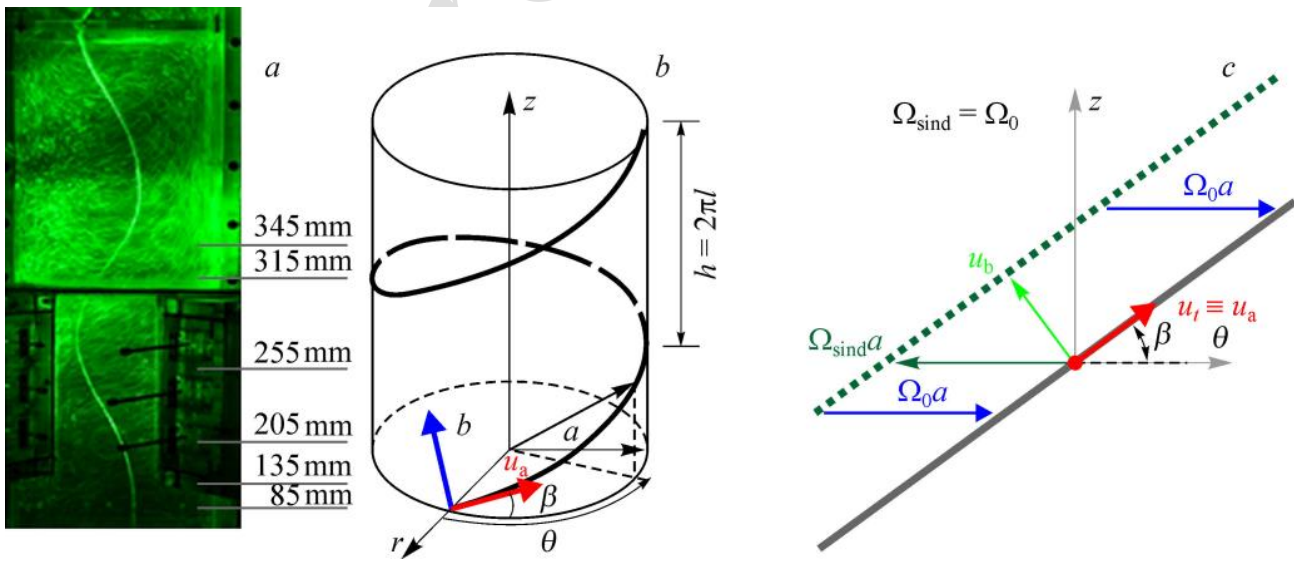

Fig. 1. Visualization of a stationary helical vortex $[15,16](a)$ diagram of absolute motion of particles along the vortex axis (thick line) preserves the stationarity $(b)$ and cylindrical representation of the helical vortex with its imaginary position (dotted line) acquired through a self-induced shift $\Omega_{\text {sind }} a$, which would take place in infinite space, and return to a stationary position (solid line) due to the compensating speed $\Omega_{0} a$, induced by the walls of the experimental chamber $(c)$.

$a$ - radius of the helical vortex, $h=2 \pi l$ - helical pitch, $\beta$-inclination angle of helical line relative to horizontal plane, $(r, \theta, z)$ - cylindrical coordinates; $u_{\mathrm{b}}$ — self-induced velocity (directed along binormal); $u_{\mathrm{a}}$ - absolute velocity of a fluid particle, coinciding for a stationary vortex with the tangential component $u_{\mathrm{t}}$. 

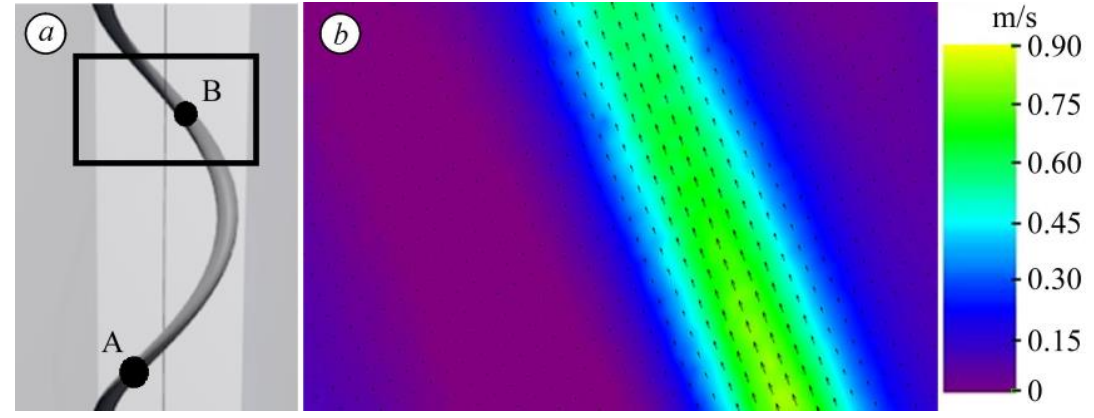

Fig. 2. The vector velocity field in a vertical section passing through the axis of the vortex.

$a$ - measurement area and reference points on the vortex axis; $b$ - vectors and contours of velocity (the direction of particle velocity is seen to coincide with the vortex axis).

the motion of helical vortices contains only the motion of fluid particles, for which the absolute helical motion along the axis of the vortex is natural, but this cannot be a proof of the motion of the vortex itself. The disadvantage of the works $[12,13]$ is that they associate the movement of particles with that of three-dimensional vortex filaments, which leads to an incorrect assessment of the velocity of the filaments (Fig. 1c). The experiment conducted in this paper proves that the main assumption in $[12,13]$, expressed in the identification of the motion of helical vortices with that of fluid particles along their axis, is erroneous.

\section{On approximating the helical vortex velocity with the use of a velocity field induced by an infinitely thin filament}

The next important question is related to the possibility of approximating the velocity of helical vortices. It may seem that in $[12,13]$ a new algorithm was proposed for determining the axial and azimuthal velocities on the axis of the vortex of a finite section using a half-sum of velocities, induced by an infinitely thin vortex filament at diametrically opposite points on the surface of the vortex (equations 2.8 and 2.9 from [12]). When evaluating the novelty of this "proposal", it should be taken into account that this representation through the half-sum of velocities is well known and was previously studied for the binormal component of velocity (see, for example, [6-8]). In the cited works, the axial and azimuthal components and their half-sums were used, but only for calculating the regular remainder $C_{\mathrm{H}}$ in the asymptotic expansion of the binormal velocity component in the vicinity of an infinitely thin vortex filament (see, for example, section 4.1 in [6]).

As for the velocity of the vortex motion, in these works it was found after comparing the asymptotic representation for the induced binormal velocity at a distance $\varepsilon$ from the vortex axis with the formula for the self-induced velocity of the vortex with the core radius $\varepsilon$, proposed in [5]. Thus, the difference between the analysis of $[12,13]$ from that set out in [6-8], is

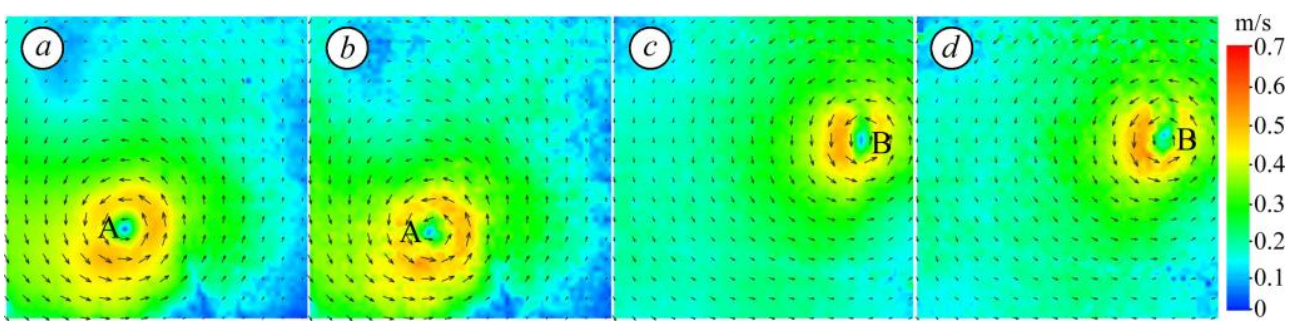

Fig. 3. Velocity field (color gradation) at different time moments in two different horizontal sections. $\mathrm{A}(a, b)$ and $\mathrm{B}(c, d)$ - points on the vortex axis that remain stationary within the measurement error (see figure 2). 

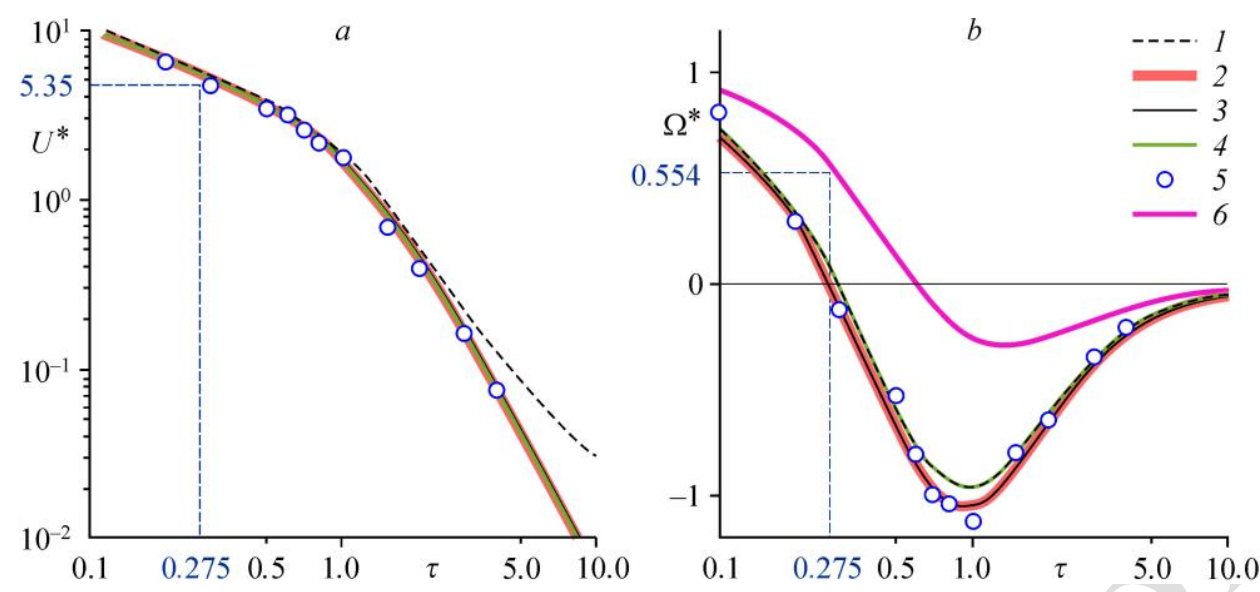

Fig. 4. Graphs of axial ( $a$ ) and angular $(b)$ displacements of a fluid particle along the axis of a helical vortex with a finite core of radius of 0.1 , depending on the dimensionless pitch $\tau$.

The dimensionless values $U^{*}$ and $\Omega^{*}$ refer to the absolute velocity coordinates and are introduced through the ratio $\Gamma / 4 \pi a$ for translational velocity and through $\Gamma / 4 \pi a^{2}$ for angular velocity;

1 - calculation in the absolute coordinate system using formulas from [8], 2-5 are reproduced from work [12]; 2 - calculation by half-sum at a distance of 0.1 from the singular filament; 3 - calculation with discretization of a vortex with a finite core by singular filaments,

4 - calculation using the approximate Rosenhead-Moore formula, 5 - numerical solution of Euler equations); 6 - calculation in the moving coordinate system from [8].

as follows. In the first case, the half sum of the axial and azimuthal velocities in both points is found first, and then, for example, the binormal one is calculated. In the second case, the binormal velocity is presented in each point via axial and azimuthal velocities and then their half-sum is calculated. This obvious tautology, of course, gives a complete coincidence of the velocities of the solution (2.17) in [12] with the solution of [7] with constant $C_{\mathrm{H}}$ for an infinitely thin vortex filament. It should be noted that the "solution" from [12] for each component was written using the integral representation of Kapteyn series from [7], with separating the singularity and representing the regular remainder in an integral term, whose equivalence was exactly proved analytically. Further, the singularities in the Kapteyn series were also separated out in the analytical form [18, 19]. In addition, in [20], the equivalence between representations of the solution via the Biot-Savart integral and Kapteyn series was analytically proved. Therefore, even if the tautology was derived due to a mistake in [12], the comparison with the exact solution for the $C_{\mathrm{H}}$ from [7] is sufficient for its verification. Ref. [12] has a further unclear step, namely, numerical confirmation of the "solution".

Despite the availability of highly accurate numerical data of [7] for the half-sum of velocities induced by an infinitely thin filament (the case with $C_{\mathrm{H}}$ ), in [12] it was again numerically tested using several numerical approximations. These were the integral Helmholtz equation with discretization of the finite core by a hundred infinitely thin filaments, approximated by finite segments of broken curves, the approximation for the Biot-Savart law made by Rosenhead-Moore for the vortex ring (see, for example, [21]) with correction for the finite size of the vortex core, and direct numerical calculation of the Euler equations. All calculations demonstrated a varying difference from the tested solution (Fig. 4). The differences were not explained, and the results of the influence of the core discretization method, the selection of the adjusted parameter or the grid in the calculations were not given. On the basis of comparisons made after evaluating the velocity values at two diametrically opposite points on the boundary of the core, and calculation of their half-sum, it was concluded that the vortex velocity can be calculated for the case of a uniform distribution of vorticity in the vortex core. 
Fig. 5. Sketch of helical vortex displacement in the presence of only axial motion (along the axis of the chamber) of fluid particles with velocity $u_{\mathrm{a}}$.

It was argued that it is how "the actual velocity of the fluid particles lying on the centreline, i.e. the velocity of the vortex" is found. But this conclusion, although to a much lesser extent than the first one, is also incorrect. [12] failed to set the difference between the solutions for the self-induced velocity of helical vortex with a finite core and the approximation of the solution by the half-sum of velocities, induced by a singular helical filament at finite distance from it equal to the core radius. However, the difference between the two solutions, although not very significant, does exist. Previously, it was es-

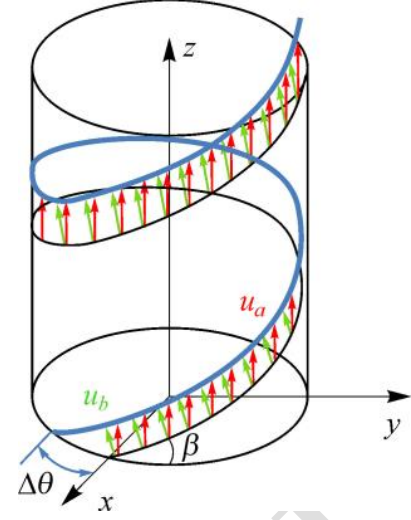
tablished numerically in [6] and analytically in [7] to correspond to a difference of 1/4 between the regular $C_{\mathrm{H}}$ and $C_{\mathrm{MS}}$ remainders, where the latter is derived by the Moore-Saffman (MS) [21] procedure for a curved vortex with a uniform core. Both reminders were introduced after the same normalization and separation of summands corresponding to the pole and logarithm in the singular solution. It turns out that the calculations in [12], arising in that 24 years, were made with more errors than in [6] and the accurate solutions of [7] were also ignored.

\section{The invariance of motion in the inertial reference system}

The equivalence of motion of helical vortices in different inertial coordinate systems will be shown to justify the universality of the difference in these motions shown experimentally above. We do this by comparing the two curves on the right fragment of figure 5 in [12] (the red and green lines of this figure correspond to the red and pink lines in figure $4 b$ of this work), the difference of which should supposedly indicate an error made in [8]. This comparison itself is the worst error of [12], since the curves were obtained in different coordinate systems. In [12] a helical vortex in a stationary system is considered, and on pages 328 and 335 in [8] it is indicated that a system consisting of $N$ helical vortices of the same strength $\Gamma$ translates with a fixed velocity $\Gamma N / h$ where $h$ is the vortex pitch as defined in figure 1(b). The choice of a moving coordinate system in [8] was associated with providing a fixed axis in the center of the vortex multiplet to ensure an adequate comparison with the Kelvin problem on instability of a polygon of point vortices, where a fixed point was located in the center of the polygon. It is interesting to compare the red and pink curves by switching to a single coordinate system. The full comparison of the curves is difficult, since the representation of motion through the absolute velocity of particles along the axis proposed in [12] does not directly fix the new position of the vortex, in contrast to the classical consideration based on binormal velocity through the common angle of helix $\beta$. Therefore, we will correct the velocities at the point where the angle of the absolute velocity of the particles is known, i.e. when the particles move strictly in the axial direction (Fig. 5), which corresponds to zero in curve 2 in Fig. $4 b$.

The zero in the red curve in the fragment of figure 5 from [12] and in figure $4 b$ of this work corresponds to $\tau_{0} \approx 0.275$. The value of the corresponding absolute velocity at this point is $u_{\mathrm{a}}\left(\tau_{0}\right)=U^{*}\left(\tau_{0}\right) \approx 5.35$ (see fig. 4a). The angular velocity from [8] (curve 6 in Fig. 4b) has a non-zero value of $\Omega^{*}{ }_{\mathrm{Ok}}\left(\tau_{0}\right) \approx 0.554$ at $\tau_{0}=0.275$. The coordinate system for a single vortex from $[8]$ (p. 328, 335) translates with a fixed velocity $\Gamma /\left(2 \pi \mathrm{R} \tau_{0}\right)$, which corresponds to the dimensionless value $2 / \tau_{0}$. 
If we consider the motion of the coordinate system in the formula from [8], the axial velocity is given by

$$
U_{\mathrm{Ok}}=\left[\Gamma /(2 \pi a)-a \Omega_{\mathrm{Ok}}\left(\tau_{0}\right)\right] / \tau_{0}
$$

Hence, the dimensionless value $U_{\mathrm{Ok}}^{*} \approx(2-0.554) / 0.275=5.258$, which is lower than the axial velocity of the fluid particles from [12], which is equal to 5.35. The slight difference is explained by the fact that [12] ignored integration over the vortex cross section, which would correct the solution from an infinitely thin helical vortex filament. This additional correction, using the difference between $C_{\mathrm{H}}$ and $C_{\mathrm{MS}}\left(C_{\mathrm{H}}-C_{\mathrm{MS}}=1 / 4\right)$ in normalized form and the triangles in Fig. $1 c$ gives $0.25 \tau_{0} /\left(1+\tau_{0}{ }^{2}\right)^{3 / 2}=0.25 \cdot 0.275 /(1+0.075625)^{3 / 2} \approx 0.062$. Thus $5.258+$ $0.062=5.32$, which better agrees with $U^{*}\left(\tau_{0}\right)=5.35$ within the rounding errors in calculations.

Thus, the conclusion $[12,13]$ about an error in the work [8] is an incorrect one made by comparing the values determined in different coordinate systems. Comparison in the same system demonstrates their agreement. This fact can be considered as a confirmation of the general difference between the motions of the vortex and the fluid particles along the helical axis. This difference must be independent of the choice of inertial coordinate system and was established in the experiment described above.

\section{Conclusion}

The experimental study demonstrates the difference between the motion of a helical vortex in space and fluid particles moving along its axis. This difference was formulated at the beginning of the last century [4] and was successfully used in the construction of vortex rotor theory $[4,10]$. It is quite difficult to separate the two motions in the non-stationary case, since both relate to the same helical axis of the vortex. It has become possible to show the difference of these motions convincingly in the extreme case when the vortex motion was stopped in the experiment, while the fluid particles move significantly along the stationary vortex axis. The present study was motivated by the improper identification of two different motions in recent publications $[12,13]$. In short, the study has shown that 1 ) the velocity field of a non-moving helical vortex does not contain any bi-normal component; 2) the difference in the comparison in [12] of the induced velocity between the model of [8] and other models was based on a misinterpretation (moving vs non-moving frame of reference); 3 ) the technique for computing the core velocity of the helical vortex in [12] contains an error due to a missing term. Hence the study supports, in accordance with classical vortex theory but in disagreement with [12], that the motion of helical vortices is uniquely determined by the bi-normal component of the velocity.

\section{References}

1. L. Kelvin, Vibrations of a columnar vortex, Philos. Mag., 1880, Vol. 10, P. 155-168.

2. N.G. Nygaard, Wakes in very large wind farms and the effect of neighbouring wind farms, Journal of Physics: Conference Series, 2014, Vol. 524, P. 012162-1-012162-10.

3. V.L. Okulov, I.K. Kabardin, I.V. Litvinov, R.F. Mikkelsen, I.V. Naumov, J.N. Sørensen, D.H. Wood, and S.V. Alekseenko, Hydrokinetic energy conversion: the basis of hydro farm technology, in: Abs. 5th International Workshop on Heat/Mass Transfer Advances for Energy Conservation and Pollution Control August 13-16, Novosibirsk, Russia, 2019, P. 134.

4. N.E. Joukowsky, Vortex theory of screw propeller, I. Trudy Otdeleniya Fizicheskikh Nauk Obshchestva Lubitelei Estestvoznaniya, 1912, Vol. 16, Iss. 14, P. 1-31.

5. D.W. Moore and P.G. Saffman, The motion of a vortex filament with axial flow, Phil. Trans. R. Soc. Lond. A, 1972, Vol. 272, P. 403-429.

6. R.L. Ricca, The effect of torsion on the motion of a helical vortex line, J. Fluid Mech., 1994, Vol. 273, P. 241259.

7. J. Boersma and D.H. Wood, On the self-induced motion of a helical vortex, J. Fluid Mech., 1999, Vol. 384, P. 263-280. 
8. V.L. Okulov, On the stability of multiple helical vortices, J. Fluid Mech., 2004, Vol. 521, P. 319-342.

9. L.S. Da Rios, Sul moto d'un liquido indefinito con un filetto vorticoso di forma qualunque, Rendiconti del Circolo Matematico di Palermo, (On the motion of an undefined liquid with a swirling line of any shape, Reports of the Mathematical Circles of Palermo), 1906, Vol. 22, P. 117-137.

10. V.L. Okulov, J.N. Sørensen, and D.H. Wood, The rotor theories by professor Joukowsky: vortex theories, Progress in Aerospace Sci., 2015, Vol. 73, P. 19-46.

11. G.K. Batchelor, An Introduction to Fluid Dynamics, Cambridge University Press, Cambridge, 1973.

12. O.V. Fuentes, Motion of a helical vortex, J. Fluid Mech., 2018, Vol. 836, R1.

13. O.V. Fuentes, Flow topology of helical vortices, J. Fluid Mech., 2018, Vol. 842, R2.

14. V.L. Okulov and J.N. Sørensen, The self-induced motion of a helical vortex, J. Fluid Mech., 2020, Vol. 883, A-5.

15. S.V. Alekseenko, P.A. Kuibin, V.L. Okulov, and S.I. Shtork, Helical vortices in swirl flow, J. Fluid Mech., 1999, Vol. 382, P. 195-243.

16. E.S. Gesheva, I.V. Litvinov, S.I. Shtork, and S.V. Alekseenko, Analyzing the aerodynamic structure of swirl flow in vortex burner models, Thermal Engineering, 2014, No. 9, P. 649-657.

17. S.I. Shtork, E.S. Gesheva, P.A. Kuibin, V.L. Okulov, and S.V. Alekseenko, Parametric description of the stationary helical vortex in a hydrodynamic vortex chamber. J Appl Mech Tech Phys 2020, Vol. 61, P. 359-367.

18. V.L. Okulov, The velocity field induced by vortex filaments with cylindrical and conic supporting surface, Russian J. Engng Thermophys., 1995, Vol. 5, P. 63-75.

19. P.A. Kuibin and V.L. Okulov, Self-induced motion and asymptotic expansion of the velocity field in the vicinity of a helical vortex line, Phys. Fluids, 1998, Vol. 10, P. 607-614.

20. J.C. Hardin, The velocity field induced by a helical vortex filament, Phys. Fluids, 1982, Vol. 25, P. 1949-1952.

21. P.G. Saffman, Vortex Dynamics, Cambridge University Press, Cambridge, 1995. 